\title{
Topological confinement in trilayer graphene
}

\author{
S. H. R. de Sena, ${ }^{1, *}$ J. M. Pereira Jr., ${ }^{2, \dagger}{ }^{\dagger}$ F. M. Peeters,${ }^{3,2, \ddagger}$ and G. A. Farias ${ }^{2, \S}$ \\ ${ }^{1}$ Universidade da Integração Internacional da Lusofonia Afro-Brasileira, Campus da Liberdade, Redenção, Ceará, 62790-000, Brazil \\ ${ }^{2}$ Departamento de Física, Universidade Federal do Ceará, Fortaleza, Ceará, 60455-760, Brazil \\ ${ }^{3}$ Department of Physics, University of Antwerp, Groenenborgerlaan 171, B-2020 Antwerpen, Belgium \\ (Received 4 September 2013; revised manuscript received 2 January 2014; published 16 January 2014)
}

\begin{abstract}
We calculate the spectrum of states that are localized at the interface between two regions of opposite bias in trilayer graphene (TLG). These potential profiles, also known as potential kinks, have been predicted to support two different branches of localized states for the case of bilayer graphene, and show similarities to the surface states of topological insulators. On the other hand, we found that ABC stacked TLG exhibits three different unidimensional branches of states in each valley that are confined to the kink interface. They have the property $E\left(k_{y}\right)=-E\left(-k_{y}\right)$ when belonging to the same valley and $E_{K}\left(k_{y}\right)=-E_{K^{\prime}}\left(-k_{y}\right)$. A kink-antikink potential profile opens a gap in the spectrum of these one-dimensional states.
\end{abstract}

DOI: 10.1103/PhysRevB.89.035420

PACS number(s): 73.21.Hb, 71.10.Pm, 81.05.ue

\section{INTRODUCTION}

Graphene, a single atomic layer of hexagonal carbon, has been the focus of intense research, since the discovery of methods for its production [1,2]. This effort has also led to a growing interest in the study of few-layer graphene, in particular of bilayer and trilayer graphene. The important features of few-layer graphene arise from the coupling between the layers, which can considerably change the band structure of the system. In the case of bilayer graphene (BLG), the spectrum has been found to develop a gap if an asymmetry between the upper and lower layers is introduced. This asymmetry can be produced by doping and also by external gates which create an electric field perpendicular to the plane of the sample [3]. Thus, BLG can be pictured as a semiconductor with a tunable gap, which is a property that can be exploited for the development of devices, such as quantum dots [4], and quantum rings [5]. One feature of such gaps is that they do not depend on the sign of the bias, but only on its strength. Using this fact, Martin et al. [6] proposed a setup in which the interface between two regions with electric fields of the same magnitude but opposite direction allows the confinement of midgap states which propagate along the interface between the two regions [7] [see Fig. 1(b)]. The existence of these confined states is related to the discontinuous change in topological charge between the two regions [8,9], which can also be induced by an interlayer stacking reversal [10]. A similar mechanism is responsible for the existence of gapless surface states on topological insulators. Thus, this particular kind of potential profile creates a one-dimensional conducting channel in an otherwise semiconducting medium. Furthermore, the current in these channels is predicted to be valley polarized, i.e., electrons with different valley index propagate in opposite directions.

In comparison with single layer and bilayer graphene, trilayer graphene (TLG) introduces a new aspect, namely its spectrum depends strongly on the type of stacking of the layers.

\footnotetext{
*silviahelena@ fisica.ufc.br

†pereira@fisica.ufc.br

${ }^{\ddagger}$ francois.peeters@uantwerpen.be

§gil@ fisica.ufc.br
}

The two most important stacking orders are the rhombohedral or $\mathrm{ABC}$ stacking, and the Bernal or ABA stacking. In the absence of bias, the electronic spectrum at low energies for the ABA case can be approximately understood as a superposition of the dispersion curves of single layer and bilayer graphene, viz. a band with linear dispersion $(\omega \propto k)$ and a gapless parabolic band $\left(\omega \propto k^{2}\right)$. For the $\mathrm{ABC}$ case, on the other hand, for energies close to the Dirac point, the spectrum shows a $k^{3}$ dependence at low momenta [11]. In addition, in the presence of bias, the spectrum of ABA TLG has been shown to develop a small overlap between the valence and the conductance bands at low momenta, whereas for ABC TLG the presence of bias induces an energy gap [12-15]. Thus, in analogy with the prediction of topological confined states in bilayer graphene, it will be interesting to investigate whether the presence of a kink potential can also cause the appearance of midgap states in TLG, and whether the number of such states is now larger. Therefore, in this work we investigate the spectrum of TLG with a potential profile that displays a sharp inversion along one direction. Such potential kinks can be created by the use of split gates defined on the surface of the sample using, e.g., lithographic techniques.

The paper is organized as follows: Sec. II gives a description of the model. In Sec. III we show and discuss the analytical and numerical results. A summary and our conclusions are presented in Sec. IV.

\section{ABC STACKING: SPECTRUM}

We consider the case of ABC-stacked TLG which, within a nearest-neighbor continuum approach can be described by the Hamiltonian

$$
\mathcal{H}_{v}=\left(\begin{array}{cccccc}
U_{1} & v_{F} \pi^{\dagger} & \gamma_{1} & 0 & 0 & 0 \\
v_{F} \pi & U_{1} & 0 & 0 & 0 & 0 \\
\gamma_{1} & 0 & U_{2} & v_{F} \pi & 0 & 0 \\
0 & 0 & v_{F} \pi^{\dagger} & U_{2} & 0 & \gamma_{1} \\
0 & 0 & 0 & 0 & U_{3} & v_{F} \pi^{\dagger} \\
0 & 0 & 0 & \gamma_{1} & v_{F} \pi & U_{3}
\end{array}\right),
$$



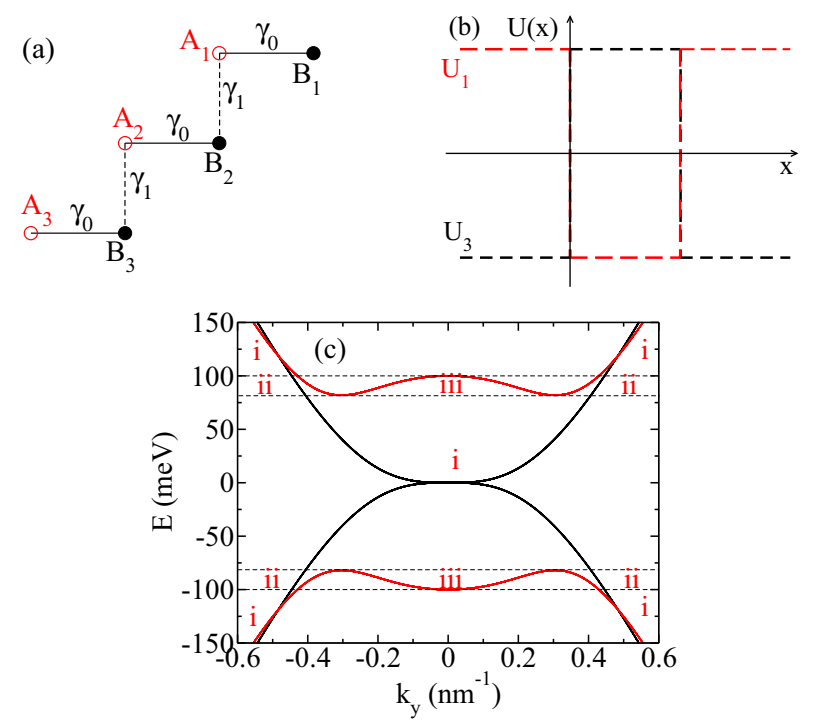

FIG. 1. (Color online) (a) Diagrammatic scheme of couplings in graphene trilayers for $\mathrm{ABC}$ stacking. (b) Example of potential profile applied to the bottom and upper layers that support chiral bound states. (c) TLG spectrum with no bias (black) and with bias (red), where $U_{2}=0, U_{1}=-U_{3}=100 \mathrm{meV}$.

whose eigenstates are defined by the six components spinor $\Psi=\left[\begin{array}{llllll}\psi_{A_{1}} & \psi_{B_{1}} & \psi_{B_{2}} & \psi_{A_{2}} & \psi_{A_{3}} & \psi_{B_{3}}\end{array}\right]^{T}$, and $v=1(-1)$ for $K$ $\left(K^{\prime}\right)$ valley. In the above Hamiltonian $\pi \equiv p_{x}+v i p_{y}, \pi^{\dagger} \equiv$ $p_{x}-v i p_{y}, v_{F}=3 a \gamma_{0} /(2 \hbar)$ is the Fermi velocity in terms of the in-plane nearest neighbor hopping $\gamma_{0}=3.12 \mathrm{eV}$, and the carbon-carbon distance $a=1.42 \AA . U_{1,2,3}$ is the potential in each layer, and $\gamma_{1}=0.4 \mathrm{eV}$ is the nearest neighbor coupling term between adjacent layers. In this model, the electron states in TLG are obtained by solving the following six coupled differential equations:

$$
\begin{aligned}
\mathcal{A}^{+} \phi_{B_{1}}+i \gamma_{1}^{\prime} \phi_{B_{2}} & =i\left(\epsilon-u_{1}\right) \phi_{A_{1}}, \\
\mathcal{A}^{-} \phi_{A_{1}} & =i\left(\epsilon-u_{1}\right) \phi_{B_{1}}, \\
\mathcal{A}^{-} \phi_{A_{2}}+i \gamma_{1}^{\prime} \phi_{A_{1}} & =i\left(\epsilon-u_{2}\right) \phi_{B_{2}}, \\
\mathcal{A}^{+} \phi_{B_{2}}+i \gamma_{1}^{\prime} \phi_{B_{3}} & =i\left(\epsilon-u_{2}\right) \phi_{A_{2}}, \\
\mathcal{A}^{+} \phi_{B_{3}} & =i\left(\epsilon-u_{3}\right) \phi_{A_{3}}, \\
\mathcal{A}^{-} \phi_{A_{3}}+i \gamma_{1}^{\prime} \phi_{A_{2}} & =i\left(\epsilon-u_{3}\right) \phi_{B_{3}},
\end{aligned}
$$

where we have used $\psi_{A(B)_{j}}(x, y)=e^{i k_{y} y} \phi_{A(B)_{j}}(x)$, since $\left[p_{y}, \mathcal{H}_{\nu}\right]=0$ for $U_{j}$ constant in the $y$ direction, and defined $\gamma_{1}^{\prime}=\gamma_{1} / \hbar v_{F}, \epsilon=E / \hbar v_{F}, u_{j}=U_{j} / \hbar v_{F}$, and $\mathcal{A}^{ \pm}=\frac{d}{d x} \pm k_{y}$.

Assuming that the gate potentials $U_{j}$ are constant we assume plane wave solutions which result in the six components spinor

$$
\begin{aligned}
& \phi_{A_{1}}=\sum_{j=1}^{3}\left(A_{j} e^{i k_{j} x}+B_{j} e^{-i k_{j} x}\right), \\
& \phi_{B_{1}}=\sum_{j=1}^{3} \frac{1}{\delta_{1}}\left(g_{j} A_{j} e^{i k_{j} x}-f_{j} B_{j} e^{-i k_{j} x}\right),
\end{aligned}
$$

$$
\begin{aligned}
\phi_{B_{2}} & =\sum_{j=1}^{3} \frac{p_{j}}{\gamma_{1}^{\prime} \delta_{1}}\left(A_{j} e^{i k_{j} x}+B_{j} e^{-i k_{j} x}\right), \\
\phi_{A_{2}} & =\sum_{j=1}^{3} \frac{r_{j}}{\gamma_{1}^{\prime} \delta_{1}}\left(\frac{A_{j}}{g_{j}} e^{i k_{j} x}-\frac{B_{j}}{f_{j}} e^{-i k_{j} x}\right), \\
\phi_{A_{3}} & =\sum_{j=1}^{3} \frac{s_{j}}{\gamma_{1}^{\prime 2} \delta_{1} \delta_{3}}\left(\frac{f_{j}}{g_{j}} A_{j} e^{i k_{j} x}+\frac{g_{j}}{f_{j}} B_{j} e^{-i k_{j} x}\right), \\
\phi_{B_{3}} & =\sum_{j=1}^{3} \frac{s_{j}}{\gamma_{1}^{\prime 2} \delta_{1}}\left(\frac{A_{j}}{g_{j}} e^{i k_{j} x}-\frac{B_{j}}{f_{j}} e^{-i k_{j} x}\right),
\end{aligned}
$$

where $\delta_{j}=\epsilon-u_{j}, \quad g_{j}=k_{j}+i k_{y}, \quad f_{j}=k_{j}-i k_{y}, \quad p_{j}=$ $\delta_{1}^{2}-\left(k_{j}{ }^{2}+k_{y}{ }^{2}\right), q_{j}=\delta_{2}{ }^{2}-\left(k_{j}{ }^{2}+k_{y}{ }^{2}\right), r_{j}=\delta_{2} p_{j}-\gamma_{1}^{\prime 2} \delta_{1}$, $s_{j}=p_{j} q_{j}-\gamma_{1}^{\prime 2} \delta_{1} \delta_{2}$. The wave vectors $k_{j}$ are the solutions of the cubic equation:

$$
\left(k_{j}^{2}+k_{y}{ }^{2}\right)^{3}-b\left(k_{j}^{2}+k_{y}^{2}\right)^{2}+c\left(k_{j}^{2}+k_{y}^{2}\right)-d=0,
$$

with

$$
\begin{aligned}
& b=\delta_{1}^{2}+\delta_{2}^{2}+\delta_{3}^{2}, \\
& c=\delta_{1}^{2} \delta_{2}^{2}+\delta_{1}^{2} \delta_{3}^{2}+\delta_{2}^{2} \delta_{3}^{2}-\gamma_{1}^{\prime 2} \delta_{2}\left(\delta_{1}+\delta_{3}\right), \\
& d=\left(\delta_{1} \delta_{2} \delta_{3}\right)^{2}-\gamma_{1}^{\prime 2} \delta_{1} \delta_{2} \delta_{3}\left(\delta_{1}+\delta_{3}\right)+\gamma_{1}^{\prime 4} \delta_{1} \delta_{3} .
\end{aligned}
$$

\section{KINK POTENTIAL PROFILE}

Let us now consider the sharp kink potential [see Fig. 1 (b)] applied to the layers, where we take $U_{2}=0$ and

$$
U_{1}=-U_{3}=\left\{\begin{array}{l}
\left.U_{0}, x<0 \text { (region } \mathrm{I}\right) \\
-U_{0}, x>0 \text { (region II). }
\end{array}\right.
$$

From now on, we will refer to a kink every time the electric polarization on the layers changes in a way that makes the electric field flip from $-\mathcal{E} \hat{z}$ to $\mathcal{E} \hat{z}$, and we call antikink when the electric field flips from $\mathcal{E} \hat{z}$ to $-\mathcal{E} \hat{z}$. This kind of bias between the bottom and top layers opens a gap in the electronic spectrum [16] as shown by the red curves in Fig. 1(c). We are interested in solutions that describe localized states along the domain wall that separates the different regions of the potential. It can be numerically checked that inside the energy gap, we have three types of regions [see Fig. 1(c)]: Inside region $i$ we have $k_{1}=i \beta_{1}, k_{2}=\alpha_{2}+i \beta_{2}$, and $k_{3}=\alpha_{2}-i \beta_{2}$ with $\alpha_{j}, \beta_{j}>0$, then in order to have a well-behaved wave function we need to set $A_{1}^{I}=A_{2}^{I}=B_{3}^{I}=B_{1}^{I I}=B_{2}^{I I}=A_{3}^{I I}=0$, where $I(I I)$ stands for $x<0(x>0)$; in region $i i$ we have $k_{1}=i \beta_{1}$, $k_{2}=i \beta_{2}$ and $k_{3}=i \beta_{3}$, and therefore in this region we have to set $A_{1}^{I}=A_{2}^{I}=A_{3}^{I}=B_{1}^{I I}=B_{2}^{I I}=B_{3}^{I I}=0$; in region $i$ ii of the spectrum, we find two real wave vectors, and therefore no confined states are obtained in this region. In order to avoid any misunderstanding, we emphasize that the curves in Fig. 1(c) correspond to a cross section, with $k_{x}=0$, of the actual spectrum $E\left(k_{x}, k_{y}\right)$. Since the minimum of the conduction band and the maximum of the valence band occur at $k_{x}=0$, Fig. 1(c) help us to visualize the range of the energy gap, identified by the region $i$ in the figure. Furthermore, due to the peculiar "mexican hat" shape of the spectrum in the biased case (red 

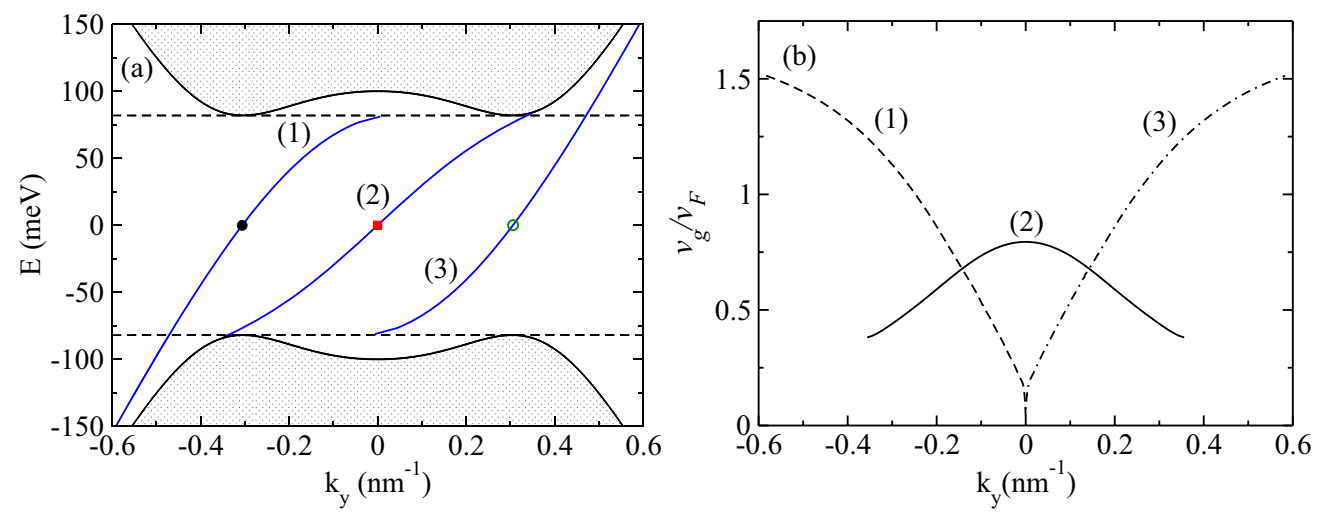

FIG. 2. (Color online) (a) The blue curves are the electronic states for the $K$ valley that are localized at sharp kink for $U_{0}=100 \mathrm{meV}$, while the black curves delimit the cross-hatched free particle regions. (b) The group velocity for the energy branches which are indicated by the numbers (1), (2), and (3) in panel (a).

curves), the region $i$ ii, just below the "mexican hat," supports propagating states for nonzero $k_{x}$, which explains the fact that no confined states can be found in that region.

Apart from the single kink potential, another profile of interest is the one that consists of a sequence of kinks and antikinks. In the general case, where the potential profile is a sequence of kinks and antikinks, we have to rely on a numerical approach. The transfer matrix method relates the wave function coefficients in the first and last potential regions $n$ as follows

$$
\left(\begin{array}{l}
A_{1}^{n} \\
B_{1}^{n} \\
A_{2}^{n} \\
B_{2}^{n} \\
A_{3}^{n} \\
B_{3}^{n}
\end{array}\right)=\mathcal{N}\left(\begin{array}{l}
A_{1}^{1} \\
B_{1}^{1} \\
A_{2}^{1} \\
B_{2}^{1} \\
A_{3}^{1} \\
B_{3}^{1}
\end{array}\right)
$$

where $\mathcal{N}$ is called the transfer matrix. By applying the previously discussed localization conditions, some of the coefficients in Eq. (7) will be zero, so that Eq. (7) can be rearranged into a determinantal equation, and the energies of the confined states can be found by solving

$$
\operatorname{det} \mathcal{M}=0 \text {, }
$$

with

$$
\mathcal{M}=\left(\begin{array}{cccccc}
-1 & \mathcal{N}_{12} & 0 & \mathcal{N}_{14} & \mathcal{N}_{15} & 0 \\
0 & \mathcal{N}_{22} & 0 & \mathcal{N}_{24} & \mathcal{N}_{25} & 0 \\
0 & \mathcal{N}_{32} & -1 & \mathcal{N}_{34} & \mathcal{N}_{35} & 0 \\
0 & \mathcal{N}_{42} & 0 & \mathcal{N}_{44} & \mathcal{N}_{45} & 0 \\
0 & \mathcal{N}_{52} & 0 & \mathcal{N}_{54} & \mathcal{N}_{55} & 0 \\
0 & \mathcal{N}_{62} & 0 & \mathcal{N}_{64} & \mathcal{N}_{65} & -1
\end{array}\right)
$$

for region $i$ of the spectrum and

$$
\mathcal{M}=\left(\begin{array}{cccccc}
-1 & \mathcal{N}_{12} & 0 & \mathcal{N}_{14} & 0 & \mathcal{N}_{16} \\
0 & \mathcal{N}_{22} & 0 & \mathcal{N}_{24} & 0 & \mathcal{N}_{26} \\
0 & \mathcal{N}_{32} & -1 & \mathcal{N}_{34} & 0 & \mathcal{N}_{36} \\
0 & \mathcal{N}_{42} & 0 & \mathcal{N}_{44} & 0 & \mathcal{N}_{46} \\
0 & \mathcal{N}_{52} & 0 & \mathcal{N}_{54} & -1 & \mathcal{N}_{56} \\
0 & \mathcal{N}_{62} & 0 & \mathcal{N}_{64} & 0 & \mathcal{N}_{66}
\end{array}\right)
$$

for region $i$, where $\mathcal{N}_{i j}$ are the elements of the transfer matrix, which can be easily derived.

\section{NUMERICAL RESULTS}

The three blue curves in Fig. 2(a) are the topological states that are confined at the kink interface for $U_{0}=100 \mathrm{meV}$, while the black curves delimit the cross-hatched free particle regions. The dispersion of these one-dimensional electronic states in ABC TLG are all chiral states propagating with a positive group velocity along the kink direction and are related to those previously found in BLG systems [6,7], except for the appearance of a new energy branch crossing at $\left(k_{y}=0, E=0\right)$, and for the fact that in these previous works the authors considered a simplified two-band Hamiltonian, where the Mexican hat feature is absent from the spectrum. For an antikink profile, the spectrum is simply a mirror reflection with respect to $k_{y}=0$, i.e., all the states have now a negative group velocity along the $y$ direction. In order to obtain the spectrum for electrons in the $K^{\prime}$ valley, one can repeat the same symmetry operation, i.e., a kink in the $K$ valley corresponds to an antikink in the $K^{\prime}$ valley, and vice versa. The right panel of Fig. 2 shows the group velocity for the three energy branches appearing in (a), which show a very distinct behavior from the velocities of the topological states in BLG [7]. This, together with the number of branches, are clear qualitative differences between the topological states in BLG and TLG.

Figures 3(a)-3(e) present the moduli squared of the spinor components and the corresponding probability density for those states with zero energy marked in Fig. 2(a) by symbols. Notice that Eqs. (2) are invariant under the transformation $\left(k_{y}, E\right) \rightarrow\left(-k_{y},-E\right), \phi_{B_{1}} \rightarrow i \phi_{A_{3}}, \phi_{B_{2}} \rightarrow i \phi_{A_{2}}$, and $\phi_{B_{3}} \rightarrow$ $i \phi_{A_{1}}$, which implies the same probability density for the states $\left(-k_{y}, 0\right)$ and $\left(k_{y}, 0\right)$, marked in Fig. 2(a) by the solid and open circles. In addition, it can be also concluded that $\phi_{B_{1}}=i \phi_{A_{3}}$, $\phi_{B_{2}}=i \phi_{A_{2}}$, and $\phi_{B_{3}}=i \phi_{A_{1}}$ when $k_{y}=0$ and $E=0$.

Figures 4(a) and 4(b) display the spectrum for a kinkantikink structure separated by $w=50 \mathrm{~nm}$ and $w=10 \mathrm{~nm}$, respectively. When the kink-antikink are far apart from each other, as in panel (a), the spectrum is a superposition of independent kink and antikink states, since the wave functions of such states are well localized around the domain walls 

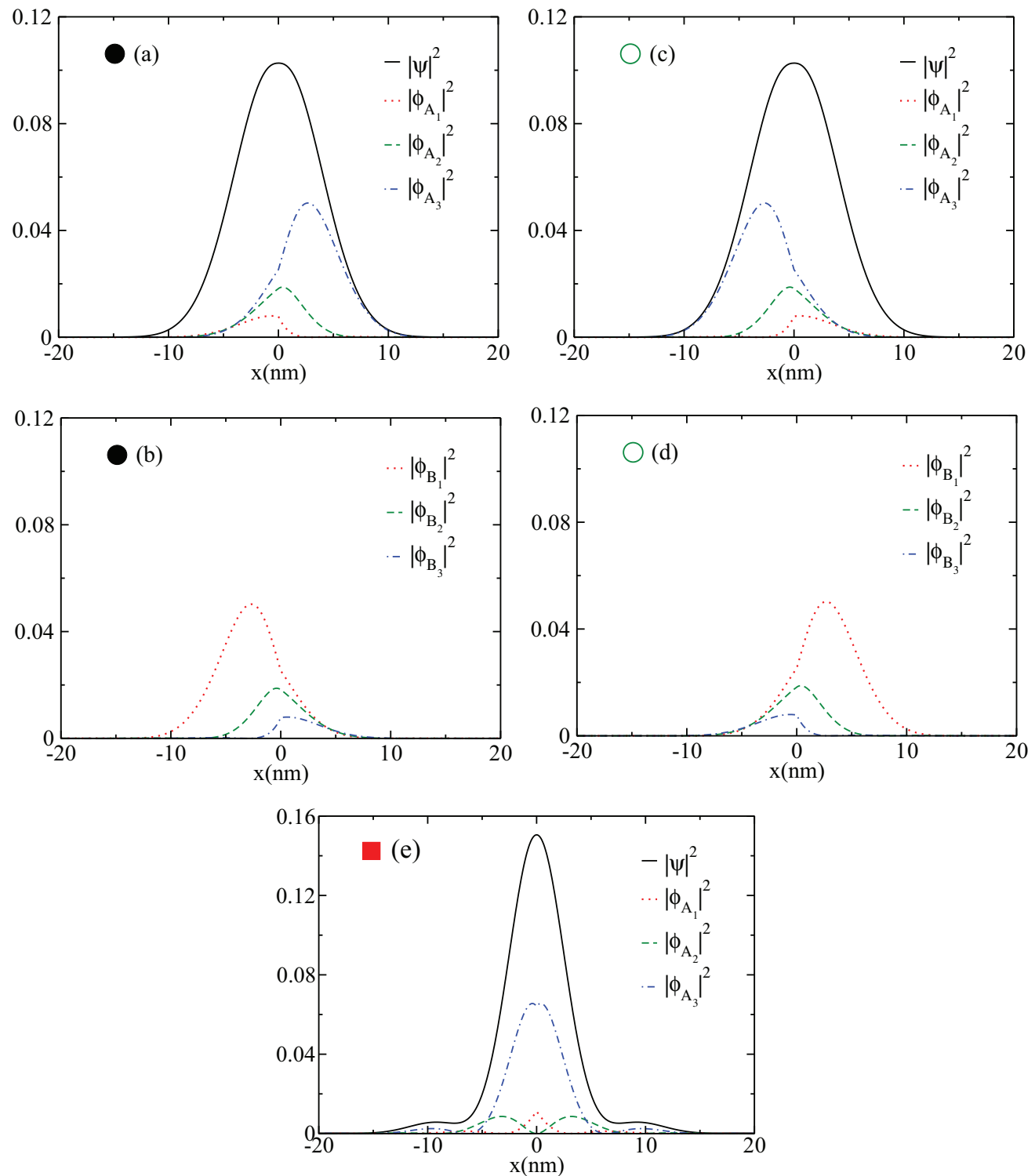

FIG. 3. (Color online) Probability density of the different spinor components for the zero energy states marked in Fig. 2(a) by symbols.

and do not interact. As the antikink approaches the kink, the states localized around these two structures start to overlap and change the spectrum, opening an energy gap and removing the $E=0$ states, as shown in panel (b).
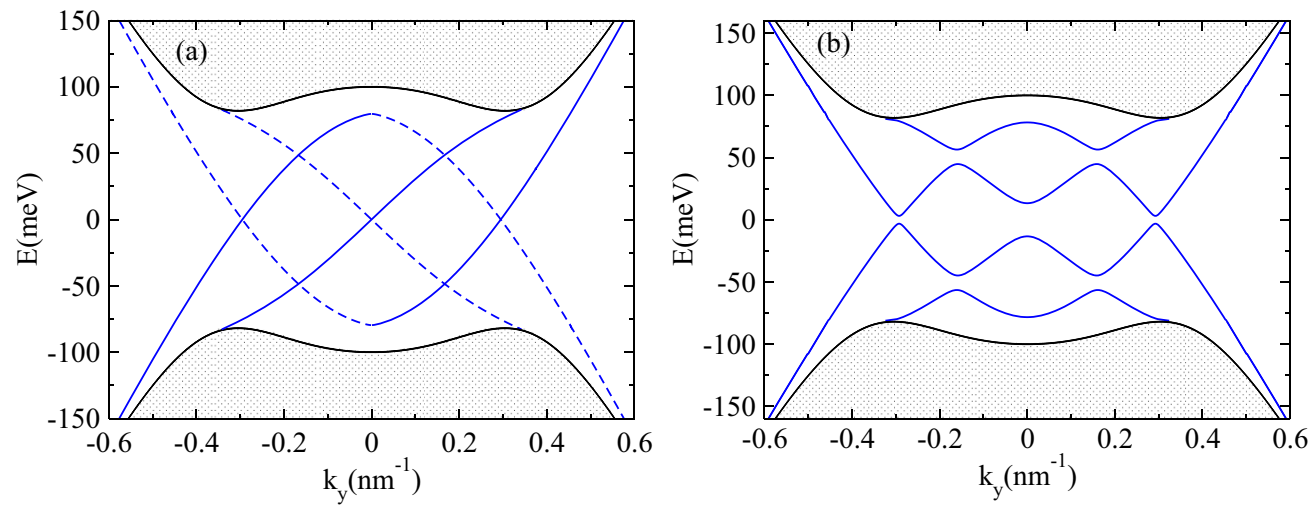

FIG. 4. (Color online) Energy spectrum around the $K$ valley for a kink-antikink potential profile with (a) $w=50 \mathrm{~nm}$ and (b) $w=10 \mathrm{~nm}$ separation between the kink and the antikink. 


\section{CONCLUSIONS}

In summary, we obtained the spectrum of localized states in trilayer graphene that arise due to the presence of potential kinks and antikinks, i.e., regions in which the perpendicular electric field applied to the structure changes sign. Similar to the case of bilayer graphene, the presence of such kinks causes the appearance of midgap states, in which the direction of propagation is valley polarized. However, in the case of ABC-stacked trilayer graphene, three topological states are found in the case of a single kink, whereas for bilayers only two states were obtained. Apart from the number of states, the dependence of the carrier velocities of those topological states on the momentum constitute another signature, helping to distinguish between BL and TLG. For potentials presenting a kink-antikink, the overlap between the states leads to the appearance of anticrossings in the spectrum, in turn creating four bands of localized states along the kink and/or antikink.
Electronic transport measurements should be able to experimentally detect these states. In the presence of such a kink potential, transport will be very anisotropic when the Fermi energy is localized in the energy gap. Along the kink direction, electronic transport will be quasiballistic while in the direction perpendicular to the kink no current will flow. However, in the presence of inhomogeneities, we expect transport as characterized by variable range hopping when the Fermi energy is this energy region.

\section{ACKNOWLEDGMENTS}

This work was supported by the Brazilian Council for Research (CNPq-PRONEX), the Flemish Science Foundation (FWO-Vl), and the Bilateral project between $\mathrm{CNPq}$ and FWO-Vl and the Brazilian program Science Without Borders $(\mathrm{CsF})$.
[1] K. S. Novoselov, A. K. Geim, S. V. Morozov, D. Jiang, Y. Zhang, S. V. Dubonos, I. V. Grigorieva, and A. A. Firsov, Science 306, 666 (2004).

[2] A. H. Castro Neto, F. Guinea, N. M. R. Peres, K. S. Novoselov, and A. Geim, Rev. Mod. Phys. 81, 109 (2009).

[3] E. McCann and V. I. Fal'ko, Phys. Rev. Lett. 96, 086805 (2006).

[4] J. M. Pereira, Jr., P. Vasilopoulos, and F. M. Peeters, Nano Lett. 7, 946 (2007); J. M. Pereira, Jr., F. M. Peeters, P. Vasilopoulos, R. N. Costa Filho, and G. A. Farias, Phys. Rev. B 79, 195403 (2009).

[5] M. Zarenia, J. M. Pereira, Jr., F. M. Peeters, and G. A. Farias, Nano Lett. 9, 4088 (2009); M. Zarenia, J. M. Pereira, Jr., A. Chaves, F. M. Peeters, and G. A. Farias, Phys. Rev. B 81, 045431 (2010).

[6] I. Martin, Ya. M. Blanter, and A. F. Morpurgo, Phys. Rev. Lett. 100, 036804 (2008).

[7] M. Zarenia, J. M. Pereira, G. A. Farias, and F. M. Peeters, Phys. Rev. B 84, 125451 (2011).

[8] J. Li, I. Martin, M. Buttiker, and A. F. Morpurgo, Nat. Phys. 7, 38 (2011).
[9] W. Yao, S. A. Yang, and Q. Niu, Phys. Rev. Lett. 102, 096801 (2009).

[10] F. Zhang, A. H. MacDonald, and E. J. Mele, PNAS 110, 10546 (2013).

[11] F. Guinea, A. H. Castro Neto, and N. M. R. Peres, Phys. Rev. B 73, 245426 (2006).

[12] A. A. Avetisyan, B. Partoens, and F. M. Peeters, Phys. Rev. B 79, 035421 (2009); 81, 115432 (2010).

[13] M. Koshino and E. McCann, Phys. Rev. B 79, 125443 (2009).

[14] S. H. Jhang, M. F. Craciun, S. Schmidmeier, S. Tokumitsu, S. Russo, M. Yamamoto, Y. Skourski, J. Wosnitza, S. Tarucha, J. Eroms, and C. Strunk, Phys. Rev. B 84, 161408(R) (2011).

[15] C. H. Lui, Z. Li, K. F. Mak, E. Cappelluti, and T. F. Heinz, Nat. Phys. 7, 944 (2011); W. Bao, L. Jing, J. Velasco, Jr., Y. Lee, G. Liu, D. Tran, B. Standley, M. Aykol, S. B. Cronin, D. Smirnov, M. Koshino, E. McCann, M. Bockrath, and C. N. Lau, ibid. 7, 948 (2011).

[16] M. Aoki and H. Amawashi, Solid State Commun. 142, 123 (2007). 